Int. J. Dev. Biol. 63: 253-258 (2019)

https://doi.org/10.1387/ijdb.190002mb

\title{
Enucleolation and nucleolus transfer in mammalian oocytes and zygotes
}

\author{
MICHAL BENC*,1,2, JOSEF FULKA, JR. ${ }^{1}$, FRANTIŠEK STREJČEK ${ }^{3}$, MARTIN MOROVIČ ${ }^{3}$, MATEJ MURÍN ${ }^{4}$, \\ STANISLAVA MARTIINKOVÁ' ${ }^{1}$, DOMINIKA JETTMAROVÁ ${ }^{1}$ and JOZEF LAURINČí|K ${ }^{2,4}$ \\ ${ }^{1}$ Institute of Animal Science, Prague, Czech Republic, ${ }^{2}$ Department of Zoology and Anthropology, Faculty of Natural \\ Sciences, Constantine the Philosopher University in Nitra, Nitra, Slovak Republic, ${ }^{3}$ Department of Botany and \\ Genetics, Faculty of Natural Sciences, Constantine the Philosopher University in Nitra, Nitra, Slovak Republic and \\ ${ }^{4}$ The Czech Academy of Sciences, Institute of Animal Physiology and Genetics, Liběchov, Czech Republic
}

\begin{abstract}
The oocyte GV/GVs (germinal vesicle/germinal vesicles) and zygot PN/PNs (pronucleus/ pronuclei) of some mammals contain clearly visible nucleoli which exhibit an atypical morphological structure. These nucleoli (NCLs) can be relatively easily manipulated, i.e. removed from GVs/ PNs or eventually transferred into another oocyte/zygote. Thus, with the help of micromanipulation techniques it was possible to uncover the real function(s) they play in processes of oocyte maturation and early embryonic development. The purpose of our review is to describe briefly the micromanipulation techniques that can be used for oocyte/zygote nucleoli manipulation. Moreover, we present some examples of results that were obtained in nucleolus manipulation experiments.
\end{abstract}

KEY WORDS: nucleolus, micromanipulation, oocyte, zygote

\section{Introduction}

The nucleoli in differentiated cells and advanced embryo developmental stages are composed of three distinct components - fibrillar, granular, dense fibrillar (Boisvert et al., 2007). On the other hand, in some mammals (mouse, horse, human, pig, etc.), fully grown oocyte nuclei (germinal vesicles), pronuclei in zygotes as well as nuclei in very early cleavage stage embryos contain very atypical NCLs that are composed exclusively of a dense fibrillar material (Tan et al., 2009; Fulka and Aoki, 2016). The function(s) of these very specific nucleoli was unknown for a very long time - it has been commonly accepted, that they may serve as a store of material(s) from which fully differentiated nucleoli are formed later on, as the embryo develops. That NCLs can be removed from fully grown oocytes or zygotes has been reported, for the first time in 2003, when they were aspirated from GVs of immature porcine oocytes - "enucleolation" (Fulka et al., 2003). Since then, the enucleolation and subsequently the NCLs transfer approaches have been the essential techniques that contributed to uncovering and understanding of the real function(s) and roles of atypical oocyte/zygote NCLs in oocyte maturation and embryo development processes (Fulka and Fulka, Jr., 2010; Fulka et al., 2011; Kyogoku et al., 2010; Fulka et al., 2015; Ogushi et al., 2008).

Moreover, the NCLs micromanipulation approaches helped us to uncover the real function that these atypical NCLs have (Fulka and Langerova, 2014; Kyogoku et al., 2014). In general, these experiments clearly indicate that these atypical NCLs serve as a major heterochromatin-organizing structure in oocytes and zygotes, at least in the mouse. In zygotes, NCLs are necessary only during very short time interval post fertilization.

Although some role(s) of atypical NCLs has been solved, many questions are not yet answered -for example: protein composition, differences between NCLs between oocytes and zygotes, species specific differences and so on (Fulka Jr. et al., IJDB - accepted). To answer these questions, the enucleolation/nucleolus transfer will be still the essential procedures.

\section{Experimental Protocols}

\section{Mouse oocytes}

Fully grown oocytes are collected either from CD1 or BDF1 (B6D2F1/Crl) mice approximately 6 -12 weeksold (Charles River). Mice are kept under standard housing conditions -12 hours light and dark cycle (light on at 8:00 a.m.), with controlled temperature,

Abbreviations used in this paper: GV, germinal vesicle; ICSI, intracytoplasmic sperm injection; MI, metaphase I; NCL, nucleolus; PN, pronucleus; PVP, polyvinylpyrrolidone; SCNT, somatic cell nuclear transfer; ZP, zona pellucida.

\footnotetext{
*Address correspondence to: Michal Benc. Institute of Animal Science. Biology of Reproduction, Pratelstvi 815, 10400, Praha 10 - Uhrineves, Czech Republic. Tel. +421915114034 - E-mail: benc.michal @gmail.com - (iD) https://orcid.org/0000-0002-1241-8266
}

Submitted: 5 January, 2019; Accepted: 21 January, 2019

ISSN: Online 1696-3547, Print 0214-6282 
humidity and air exchange. Females are injected intraperitoneally with 5-7.5 I.U. of PMSG (Intervet, Czech Republic). After about 44 hours, the animals are sacrificed by cervical dislocation, their ovaries isolated and transferred into HTF-HEPES medium (Zenith Biotech, USA) with $4 \mathrm{mg} / \mathrm{ml}$ BSA (Sigma, Czech Republic). Under the stereomicroscope at $37^{\circ} \mathrm{C}$, large antral follicles are punctured with a sharp needle. The oocytes are thus released into a medium from which they are collected with appropriate glass capillary tubes. Only fully grown oocytes with several layers of cumulus cells are used for further manipulations. Before the oocytes are manipulated, they are first cultured in MEM (Sigma, Czech Republic), supplemented with $4 \mathrm{mg} / \mathrm{ml} \mathrm{BSA}, 0.22 \mathrm{mM}$ Na-pyruvate, $50 \mu \mathrm{g} / \mathrm{ml}$ gentamicin, $150 \mu \mathrm{g} / \mathrm{ml}$ dbcAMP or $1 \mu \mathrm{M}$ Milrinone (Sigma, Czech Republic) at $37^{\circ} \mathrm{C} / 5 \% \mathrm{CO}_{2}$. After approximately $1 \mathrm{~h}$, cumulus cells are removed by vigorous pipetting with a glass pipette having approximately the same diameter as the oocyte with zona pellucida. The oocytes are then again cultured for another $3-4 \mathrm{~h}$ as above. It is our experience, that NCLs manipulation is better when the oocytes are pre-cultured for longer time.

\section{Porcine fully grown oocytes}

Porcine oocytes are obtained from slaughtered gilts in a local slaughterhouse. The ovaries are transferred to the lab in a warm saline $\left(35^{\circ} \mathrm{C}\right)$. Here, only the large antral follicles $(4-6 \mathrm{~mm}$ in diameter) are aspirated with $20 \mathrm{ml}$ syringe/22G needle. The follicular fluid is poured then on the lid of a Petri dish $(10-12 \mathrm{~cm})$ and under the stereomicroscope the large healthy oocytes are collected and transferred into a droplet of HTF-HEPES medium (Zenith Biotech, USA) supplemented with $4 \mathrm{mg} / \mathrm{ml}$ BSA (Sigma, Czech Republic). Under the stereomicroscope $\left(37^{\circ} \mathrm{C}\right)$ the oocytes are selected again and only those oocytes with several layers of cumulus cells are transferred into a culture medium - M 199 Earle's Salts (Thermo Fisher Scientific, USA) supplemented with $0.91 \mathrm{mM} \mathrm{Na-pyruvate,}$ $50 \mu \mathrm{g} / \mathrm{ml}$ Gentamicin sulfate, $1 \mathrm{mg} / \mathrm{ml} \mathrm{HEPES}, 10 \mathrm{ng} / \mathrm{ml}$ Epidermal Growth Factor, $9 \mathrm{mM} \mathrm{NaHCO}_{3}, 1 \mathrm{mM}$ L-glutamine, $10 \%$ follicular fluid, $0.57 \mathrm{mML}$-Cysteine, $1 \mathrm{IU} / \mathrm{ml}$ Luteinizing Hormone from sheep pituitary and $0.25 \mathrm{IU} / \mathrm{ml}$ Follicle Stimulating Hormone from sheep pituitary (Sigma, Czech Republic) and cultured in it at $38.5^{\circ} \mathrm{C}$ in humidified atmosphere of $5 \% \mathrm{CO}_{2}$ for $2-3 \mathrm{~h}$ before used for further manipulation. Thereafter, the cumulus enclosed oocytes are incubated in Phosphate buffered saline with $0.1 \%$ Hyaluronidase from bovine testes (Sigma, Czech Republic) and their cumulus cells are removed by gentle pipetting.

\section{Porcine growing oocytes}

Porcine growing oocytes are isolated from ovaries collected as described above. The oocytes are released from follicles of 0.6-1 $\mathrm{mm}$ in diameter. In growing oocytes, NCLs are not enclosed with a ring of heterochromatin and these oocytes do not mature in culture. For successful enucleolation, it is necessary to pre-incubate them for $24 \mathrm{~h}$ with $5 \mu \mathrm{g} / \mathrm{ml}$ actinomycin D, otherwise the GV chromatin is removed along with NCL (Kyogoku et al., 2010).

\section{Mouse one-cell stage embryos}

Mouse one-cell stage embryos can be either collected from stimulated and mated females or produced by ICSI (intracytoplasmic sperm injection). In the first case, the females are stimulated with PMSG followed by hCG injection approximately $44 \mathrm{~h}$ post PMSG. The females are then caged with males and the next day in the morning checked for the presence of vaginal plugs. Then the plug positive females are sacrified, clumps with zygotes released from ampulla into HTF-HEPES medium (Zenith Biotech, USA) supplemented with $4 \mathrm{mg} / \mathrm{ml}$ BSA (Sigma, Czech Republic) and then briefly incubated in HTF-HEPES medium (Zenith Biotech, USA) with $1 \mathrm{mg} / \mathrm{ml}$ hyaluronidase (Sigma, Czech Republic) to freed the zygotes from cumulus cells. The zygotes are then several times washed in KSOM medium (KSOMaa Zenith Biotech, USA) supplemented with $1 \mathrm{mg} / \mathrm{ml}$ BSA (Sigma, Czech Republic) and cultured in it before used. The disadvantage here is that pronuclei in naturally produced zygotes very often contain several NCLs and thus cannot be used for enucleolation.

For this reason, it is better to use ICSI produced zygotes. Moreover, in this case the staging of embryos is logically very precise. It is also our experience, that after ICSI, the incidence of zygotes with mononucleolar PNs is very high. The collection of zygotes, as well as the ICSI protocols are described in details in several excellent manuals and papers, for example by Yoshida and Perry (2007), Eppendorf Application Note 236, Hogan et al., (1994).

\section{Porcine two-cell stage parthenogenetic embryos}

Eventually, the nucleoli of another origin than from the oocytes or zygotes can be used. Kyogoku et al., (2012) replaced the porcine oocyte NCLs with nucleolar material from two-cell stage parthenogenetic embryo. As blastomere nuclei contain several nucleoli, it was necessary to incubate them first for 10 mins in HEPES -199 supplemented with cytochalasin B $(12.5 \mu \mathrm{g} / \mathrm{ml})$ and colcemide $(0.01 \mu \mathrm{g} / \mathrm{ml})$ and then to centrifuge them for 10 mins at $4000 \times \mathrm{g}$. The mini nucleoli unite and form a single large nucleolus that can be easily manipulated. It must be noted here, that this approach does not work in the mouse.

\section{Equipment}

The laboratory must be equipped for essential oocyte and embryo works, i.e., suitable $\mathrm{CO}_{2}$ incubators, Flow boxes for sterile media preparation, sterilization of instruments and glass capillaries and so on. For oocyte or embryo collections, convenient stereomicroscopes (with magnification 20x, 40x; Olympus, Nikon, Leica) with appropriate heated stage (Tokai Hit, Mini Tüb) are necessary. If pipettes are not purchased, pipette pullers and microforge (for example from Sutter/www.sutter.com/or Narishige/https://narishige-group.com/ are necessary. The manual how to prepare the suitable pipettes is enclosed with purchased equipment; eventually refer to company web pages. In our lab, the glass capillaries are purchased from Harvard Apparatus and then pulled in the lab. For holding pipettes $1.0 \mathrm{OD}$ x $0.50 \mathrm{ID}$, for injecting (enucleolation) pipettes $1.0 \mathrm{OD}$ x 0.50 ID capillaries are used.

If ready to use pipettes are purchased, these are for example from Eppendorf (holding: VacuTip 5175 108.000; injecting: Piezo Drill Tip Mouse ICSI 5175.220.005, suitable also for enucleolation).

When manipulating the nucleoli in oocytes or zygotes the most essential equipment is a good inverted microscope with Nomarski, Hoffman or another type of differential contrast. In our lab we use Olympus IX-71 equipped with Narishige micromanipulators MMO-4 (left, right side) and Eppendorf injectors CellTram vario 5176 filled with paraffin oil. The setting of the manipulation system /including Piezo/ as well as the preparation of manipulation dishes and capillaries are well described in two protocol papers (Kishigami et al., 2006; Yoshida and Perry, 2007). As for nuclear transfer or ICSI 
the use of Piezo injector greatly facilitates the enucleolation and nucleolus transfer procedure especially in the mouse. The Piezo injectors can be purchased either from Prime Tech Japan (for example type PMAS-150 or newest types) or Eppendorf PiezoXpert.

\section{Enucleolation of immature mouse or porcine oocytes}

Note: Before starting the designed experiment, it is strongly advised to do some blind experiments with spare biological material (even low quality) - oocytes and zygotes. Even when experienced in reproductive or developmental biology, it is necessary to be familiar with the arrangement of equipment (manipulator) as well as with Piezo injector.

In our lab, the enucleolation of oocytes /zygotes/ is performed in $10 \mu \mathrm{l}$ droplets of HTF-HEPES medium (Zenith Biotech, USA) supplemented with $7.5 \mu \mathrm{g} / \mathrm{ml}$ cytochalasin D (Sigma, Czech Republic) on the lid of Petri dishes (the origin of Petri dishes is not very important). When mouse oocytes are used, the manipulation medium is supplemented with milrinone or dbcAMP to prevent germinal vesicle breakdown. The droplets are covered with mineral oil (Sigma, Czech Republic). Important is a droplet(s) of 1-2 $\mu$ I PVP (polyvinylpyrrolidone). For PVP preparation see Kishigami et al., (2006) in the vicinity of a manipulation droplet as well as several droplets of manipulation media for washing the oocytes/zygotes before placing them into a final manipulation droplet. PVP serves for the injection pipette washing (inside/outside) to prevent the adhesion of aspirated biological material as well as for a smooth movement of mercury. An extra droplet for a correct arrangement of pipettes is also advised.

Whilst in mouse oocytes, the nucleolus (NCL/GV) is visible in a native state, the cytoplasm of porcine oocyte contains dark lipid droplets and this makes GV (NCL) invisible. For this reason, it is necessary to centrifuge them before manipulation. Briefly, the precultured oocytes without cumulus cells are incubated in a manipulation medium with $5 \mu \mathrm{g} / \mathrm{ml}$ cytochalasin D (Sigma, Czech Republic) for 5 mins and then centrifuged for 10 mins at $9000 \mathrm{rpm}$ $(\mathrm{RCF}=5433)$ in $0.5 \mathrm{ml}$ Eppendorf tubes. The lipid droplets move to one oocyte hemisphere (dark) whilst the other hemisphere (light) with GV is without them. This makes GV/NCL well visible. Comparison of porcine and mouse oocytes as well as the starting pipette positioning in a manipulation droplet is depicted in Fig. 1.

The oocytes $(10-15)$ used for enucleolation are several times washed in a manipulation medium and then transferred into a final manipulation droplet. Keep the oocytes in a group. The holding and injecting pipettes are positioned near this group. The selected oocyte is then firmly held in a holding pipette whilst the injection pipette is on the opposite side (Fig. $1 \mathrm{~B}, \mathrm{C}$ ). Positioning of porcine oocytes is more complicated because GV is not located centrally. To avoid the oocyte damage, sufficient perivitelline space below the site where the injection pipette penetrates zona pellucida (ZP) is very important. When the oocyte is correctly positioned move the injection pipette close to the oocyte so that the tip is in a contact with ZP. Thereafter, apply the strong intensity pulses. These pulses typically make a hole in ZP. Discard ZP plug from the pipette tip. Next, go through the hole a move slowly the pipette tip close to GV/NCL - try to push NCL!!! Apply a very mild negative pressure in the injection pipette. The nucleolus goes preferentially into the injection pipette, penetrates GV membrane and is slowly translocated into the oocyte cytoplasm - as sand in the hour glass. When NCL completely moves outside GV, the injection pipette with NCL

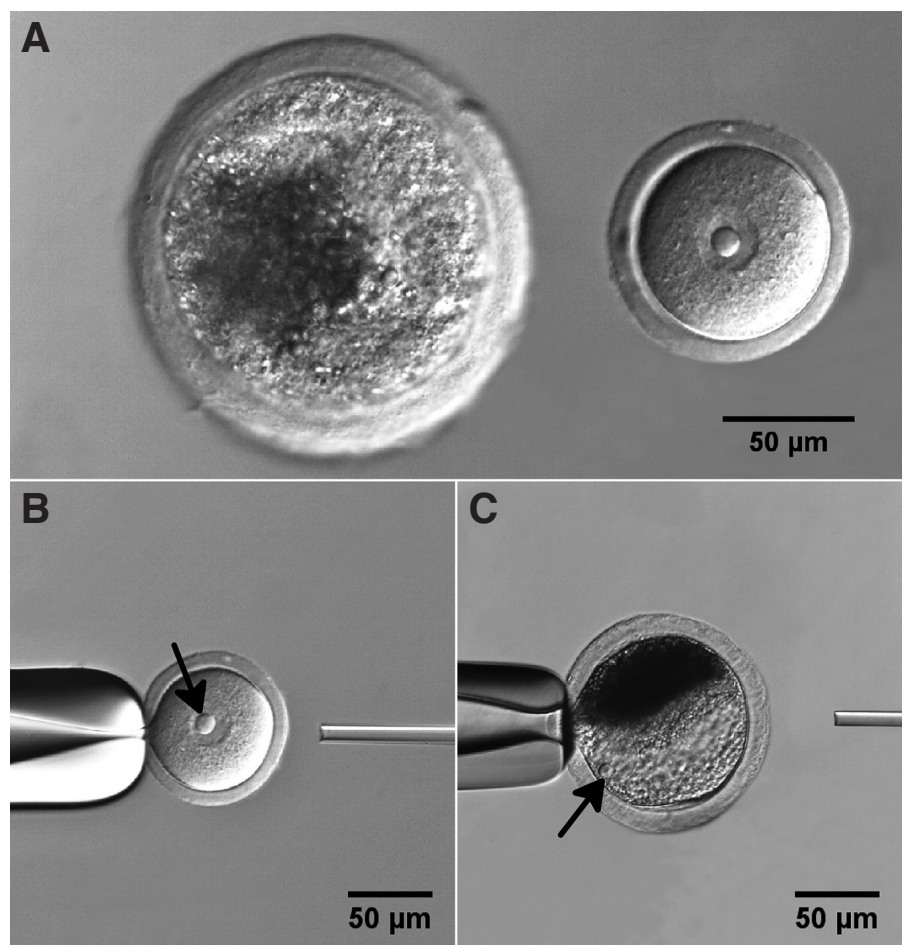

Fig. 1. Comparison of porcine and mouse oocytes. (A) Comparison of porcine (left) and mouse (right) oocytes. The porcine oocyte has been centrifuged; note the light and dark parts. The germinal vesicle (GV) is localized in the light part. The diameter of porcine oocyte is approximately $120 \mu \mathrm{m}$. Mouse oocytes is about $75 \mu \mathrm{m}$ in diameter. Its GV with nucleolus (NCL, arrow) is clearly visible without centrifugation. (B) The mouse oocyte stabilized in a holding pipette louter diameter of the mouse holding pipette is approximately $90 \mu \mathrm{m}$; inner diameter of the mouse injection pipette is approximately $10 \mu \mathrm{m})$. (C) The porcine oocyte. The holding pipette has an outer diameter approximately $90 \mu \mathrm{m}$; an inner diameter is $30 \mu \mathrm{m}$. The injection pipette is approximately $8-10 \mu \mathrm{m}$ in diameter. NCLs, arrow.

enclosed with an oocyte vitelline membrane and a small amount of the oocyte cytoplasm (nucleoloplast) is slowly withdrawn from the oocyte (Fig. 2). NCLs from nucleoloplasts can be further used for some experiments (Fulka and Langerova, 2014) or injected in another oocyte/zygote (see below).

The enucleolation of zygotes (mouse) or two-cell stage porcine embryos does not differ substantially. Here, enucleolate first one pronucleus (does not matter if male or female) or the blastomere nucleus and then the other pronucleus (blastomere nucleus).

Note: The enucleolation pipette does not penetrate into the oocyte cytoplasm. Remove NCLs from GVs very slowly, sometimes, during the enucleolation, NCL tail is formed. Wait until this tail is completely incorporated into the NCL body. When the enucleolation is done well, less than $5 \%$ of NCL material remains in GV.

\section{Long term storage of isolated nucleoli}

The isolated nucleoli can be used immediately by injecting them either into another, previously enucleolated oocyte [GV, MI (metaphase I), MII (metaphase II)] or zygote of the same or another species. Eventually, if the experimental scheme is more complicated, the NCLs must be stored. The isolated pure NCLs (not nucleoloplasts) dissolve very rapidly in a manipulation me- 


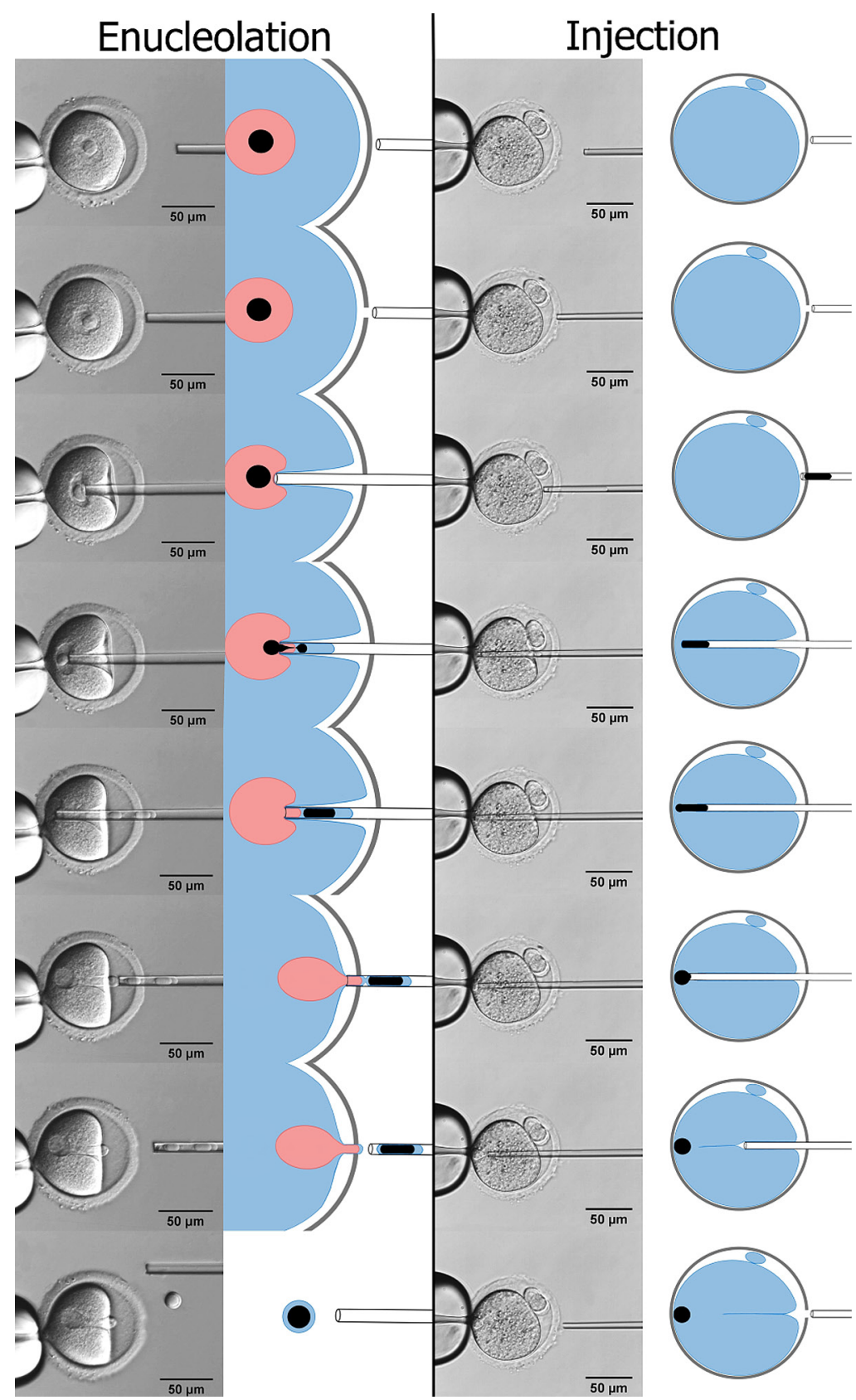

Fig. 2. Enucleolation of immature mouse oocytes and NCL transfer into mature (MII) mouse oocytes.

dium. This is not a case when NCLs are stored as nucleoloplasts. Here, the disadvantage is that nucleoplasts are difficult to handle and can be lost very easily (very small size). For this reason, it is recommended to do the designed experiments on a lid of a single petri dish with an appropriate arrangement and setting of individual different media droplets for further experiment, i.e., droplets for enucleolation, PVP droplets, droplets for transfer (Fig. 3).

In early experiments, the nucleoloplasts were put into evacuated zona pellucida and kept in a fridge $\left(4^{\circ} \mathrm{C}\right)$ in another droplet. The evacuated zonae with nucleoloplasts are well visible and it is easy to isolate NCLs from them and to use NCLs for further injection or analysis (Ogushi et al., 2008).

In a very recent study the approach of NCL storage in PVP medium has been reported (Kyogoku et al., 2018). NCLs were kept in PVP (10\%) for up to $48 \mathrm{~h}$ without losing their basic characteristics. Our experiments confirm these results. We keep the isolated pure NCLs in $12 \%$ PVP (polyvinylpyrrolidone, PVP 360) dissolved in M2 - Zenith Biotech (suggestion of Hirohisa Kyogoku, RIKEN, Kobe, JPN). Molecular weight of PVP is of critical importance. NLCs are sticky in PVP with lower molecular weight than PVP360.

\section{Nucleolus transfer}

In general, the transfer methods of NCLsare not different from SCNT (somatic cell nucleus transfer) or ICSI (Kishigami et al., 2006; Yoshida and Perry, 2007). Nevertheless, there are two main problems: first, the low quantity of NCLs; second, handling the NCLs is logically more complicated comparing to experiments where one has a "huge" quantity of material that will be injected into the oocyte (sperm, somatic cells nuclei). Whilst the transfer of oocytes into or between individual media droplets with different compositions is quite simple with no loses, the transfer of isolated NCLs (nucleoloplasts) is rather complicated because they are very tiny and can be lost quite easily. The simplest scheme is when freshly isolated nucleoli are immediately injected into another type of oocyte in the same droplet of media, for example NCLs isolated from GV staged oocytes are injected immediately into mature (MII) oocytes originating from previously enucleolated oocytes; NCLs from growing oocytes are injected into enucleolated fully grown oocytes, etc. This, however, is no always possible, because of different origin of oocytes (mouse/pig) or cell cycle staging. Here, it is better to keep the isolated NCLs in PVP and to use them later. Thus, the droplets of different media must be arranged on a lid of a Petri dish accordingly. It is our experience, that the quality of media does not change substantially when the dish is kept overnight in a fridge (see simplified droplet arrangement scheme - Fig. 3).

Moreover, it must be noted here that immature oocytes can be easily destroyed when injected with NCLs comparing to more advanced maturation stages oocytes (MI/MII). This needs certain modifications that are suitable for a given stage. For example, for GV stage oocyte injection it is better when the oocytes are kept as GV arrested in culture for about $5 \mathrm{~h}$ and the manipulation medium is supplemented with $7.5 \mu \mathrm{g} / \mathrm{ml}$ cytochalasin D (Sigma, Czech Republic). This media CD supplementation is not necessary when MI or MII oocytes are injected.

As mentioned above, the injection procedure does not differ from SCNT or ICSI. The oocyte that will be injected is stabilized with a holding pipette. If MI or MII staged, metaphase chromosomes must be located at 12 or 6 o'clock. NCL is aspirated into the injection pipette (if from PVP it is recommended to wash NCL briefly in a medium). The pipette tip then lightly touches ZP and a strong piezo pulse which makes a hole in ZP is applied. Discard ZP plug (Yoshida and Perry, 2007). Thereafter, slowly move the pipette tip into the oocyte cytoplasm so that the tip moves almost to oolemma at 9 o'clock. The NCL must be located near the pi- 


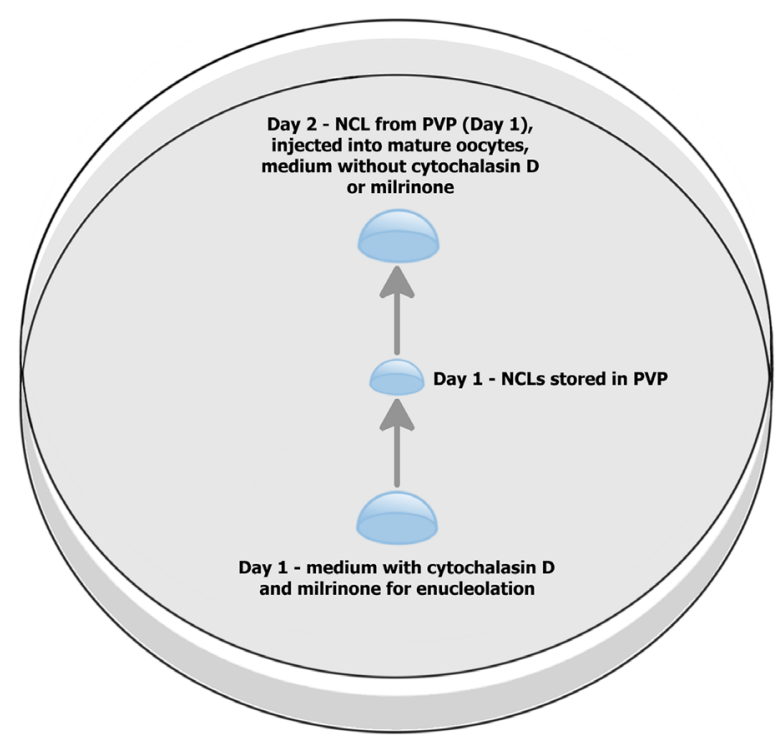

Fig. 3. Simplified arrangement of droplets on a Petri dish lid for long term storage and future manipulations of nucleoli (NCL).

pette tip. Then apply the weak piezo pulse. This pulse breaks the oolemma which is in the opening of the pipette. Gently release the NCL from a pipette and withdraw the tip slowly from the oocyte. The injected oocytes are then washed and further cultured according to the designed experimental scheme. The enucleolation and NCL transfer is shown in Fig. 2.

\section{Parthenogenetic activation}

Mouse mature (MII) oocytes are parthenogenetically activated as described by Kishigami and Wakayama in 2007. Briefly, the oocytes are incubated in KSOM medium (KSOMaa Zenith Biotech,
USA) with $0.1 \%$ BSA, $5 \mathrm{mM} \mathrm{SrCl}_{2}$ and $2 \mathrm{mM} \mathrm{EGTA} \mathrm{(Sigma,} \mathrm{Czech}$ Republic). This activation medium is supplemented either with $5 \mu \mathrm{M} /$ $\mathrm{ml}$ Latrunculin Aor $5 \mu \mathrm{g} / \mathrm{ml}$ cytochalasin B (Sigma, Czech Republic). Porcine oocytes can be activated by electric pulses. Eventually, the manipulated oocytes can be fertilized in vitro or by ICSI.

\section{Results and Discussion}

\section{Examples of NCL transfer experiments}

Nucleologenesis in parthenogenetic diploid embryos originating from NCL reinjected mouse oocytes demonstrate, how micromanipulation approaches can be used in developmental biology experiments. The mouse oocytes were enucleolated, then matured to MII and PVP stored (48h) NCLs were re-injected into them. Then these oocytes were further activated parthenogenetically and cultured for up to 5 days. The success of enucleolation was checked immunocytochemically. Nucleolar proteins B23 (Nucleophosmin/ NPM) and C23 (Nucleolin) were absent in enucleolated immature oocytes (Fig. 4)

Micromanipulation approaches are widely used in reproduction and developmental biology experiments, embryology and human assisted reproduction. With the help of these approaches, the function of atypical mammalian oocyte and zygote nucleoli has been clarified, at least in the mouse. The enucleolation and nucleolus transfer are very straightforward approaches and do not differ essentially from ICSI or SCNT. Although the role of NCL in the mouse has been to some degree clarified some questions still remain to be answered and thus the NCLs micromanipulations will play an important role in further experiments.

\section{Acknowledgments}

We thank Hirohisa Kyogoku (RIKEN, Kobe, JPN) for advising us how to preserve NCLs before he published his results.

JFJr's lab is supported from GACR 17-08605S. Further support: Slovak

Fig. 4. Localisation of nucleolar proteins B23 (Nucleophosmin/NPM) and C23 (Nucleolin). (GV) Sham-operated oocytes at GV stage. (ENU) Enucleolatedimmature oocytes - B23 and C23 are absent. Cumulus cells as positive control. (CRT) Control blastocysts originating from sham-operated oocytes with positively labelled nucleoli. (RE-INJ) Blastocysts with positively labelled nucleoli originating from NCL re-injected into previously enucleolated oocytes. Re-injected NCLs were stored for $48 h$ in $12 \%$ PVP at $4^{\circ} \mathrm{C}$. The samples were fixed and further processed exactly as described by Fulka and Langerova (2014). The labelling showed no differences between nucleologenesis in normal (non-manipulated) and NCL re-injected embryos.

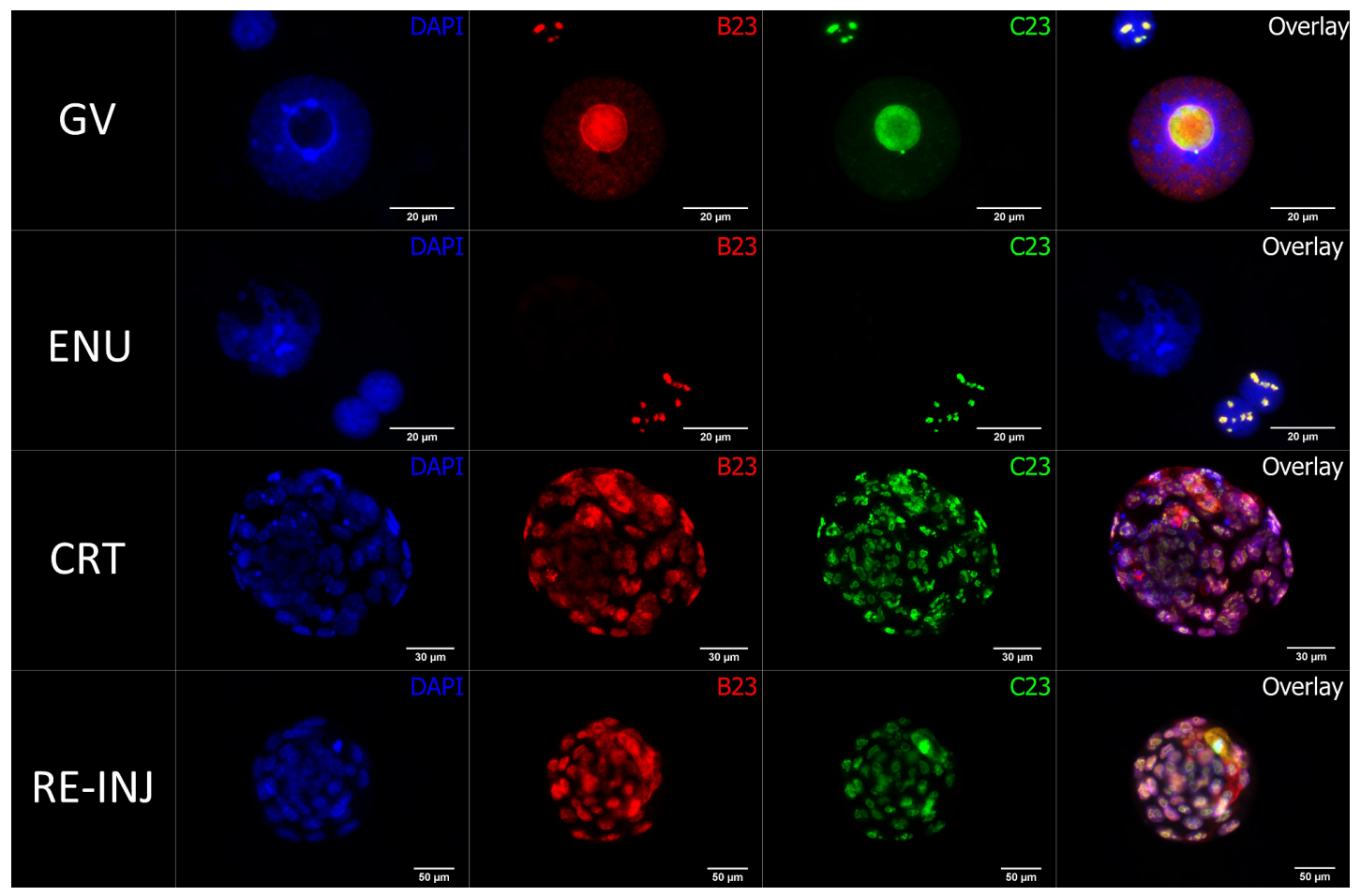


Research and Development Agency under the contract No. APVV-140001, "EXCELLENCE in molecular aspects of the early development of vertebrates", CZ.02.1.01/0.0/0.0/15_003/0000460 from the Operational Programme Research, Development and Education and by the Danish Council for Independent Research / Natural Sciences (FNU) 8021-00048B and was co-funded by the projects VEGA 1/0022/15, VEGA 1/0327/16 and 1/0001/19.

\section{References}

BOISVERT, F.M., VAN KONINGSBRUGGEN, S., NAVASCUÉS, J., LAMOND, A. I. (2007). The multifunctional nucleolus. Nat Rev Mol Cell Biol 8: 574-585.

FULKA, H., AOKI, F. (2016). Nucleolus Precursor Bodies and Ribosome Biogenesis in Early Mammalian Embryos: Old Theories and New Discoveries. Biol Reprod94:143.

FULKA, H., FULKA, J. Jr. (2010). Nucleolar transplantation in oocytes and zygotes: challenges for further research. Mol Hum Reprod 16: 63-67.

FULKA, J. Jr., LANGEROVA, A., LOI, P., MARTINKOVA, S., FULKA, H. (2011). Transplantation of nucleoli into human zygotes: not as simple as expected? $J$ Assist Reprod Genet 28: 385-389.

FULKA, H., KYOGOKU, H., ZATSEPINA, O., LANGEROVA, A., FULKA, J. Jr. (2015). Can Nucleoli Be Markers of Developmental Potential in Human Zygotes? Trends Mol Med 21: 663-672.

FULKA, H., LANGEROVA, A. (2014). The maternal nucleolus plays a key role in centromere satellite maintenance during the oocyte to embryo transition. Development 141: 1694-1704.

FULKA, J. Jr., MOOR, R.M., LOI, P., FULKA, J. (2003). Enucleolation of porcine oocytes. Theriogenology 9: 1879-1785.
HOGAN, B., BEDDINGTON, R., COSTANTINI, F., LACY, E. (1994). Manipulating the mouse embryo. Cold Spring Harbor Laboratory Press.

KISHIGAMI, S. AND WAKAYAMA, T. (2007). Efficient strontium-induced activation of mouse oocytes in standard culture media by chelating calcium. $J$ Reprod Dev 53: 1207-1215.

KISHIGAMI, S., WAKAYAMA, S., THUAN, N.V., OHTA, H., MIZUTANI, E., HIKICHI, T., BUI, H. T., BALBACH, S., OGURA, A., BOIANI, M., WAKAYAMA, T. (2006). Production of cloned mice by somatic cell nuclear transfer. Nat Protoc 1: 125-138.

KYOGOKU, H., KITAJIMA, T. S., MIYANO, T. (2014). Nucleolus precursor body (NPB) a distinct structure in mammalian oocytes and zygotes. Nucleus 5: 493-498.

KYOGOKU, H., OGUSHI, S., MIYANO, T. (2010). Nucleoli from growing oocytes support the development of enucleolated full-grown oocytes in the pig. Mol. Reprod Dev 77: 167-173.

KYOGOKU, H., OGUSHI, S., MIYANO, T. (2012). Nucleoli from two-cell embryos support the development of enucleolated germinal vesicle oocytes in the pig. Biol Reprod 87: 113.

KYOGOKU, H., WAKAYAMA, T., KITAJIMA, T. S., MIYANO, T. (2018). Single nucleolus precursor body formation in the pronucleus of mouse zygotes and SCNT embryos. PLoS One 13(8):e0202663. doi: 10.1371/journal.pone.0202663. eCollection 2018

OGUSHI, S., PALMIERI, C., FULKA, H., SAITOU, M., MIYANO, T., FULKA, J. Jr. (2008). The maternal nucleolus is essential for early embryonic development in mammals. Science 319: 613-616.

TAN, J. H., WANG, H. L., SUN, X. S., LIU, Y., SUI, H. S., ZHANG, J. (2009). Chromatin configurations in the germinal vesicle of mammalian oocytes. Mol Hum Reprod 15: 1-9.

YOSHIDA, N., PERRY, A.C. (2007). Piezo-actuated mouse intracytoplasmic sperm injection (ICSI). Nat Protoc 2: 296-304. 


\section{Further Related Reading, published previously in the Int. J. Dev. Biol.}

Faithful reprogramming to pluripotency in mammals - what does nuclear transfer teach us? Julien Maruotti, Alice Jouneau and Jean-Paul Renard Int. J. Dev. Biol. (2010) 54: 1609-1621 https://doi.org/10.1387/ijdb.103195jm

Role of the donor nuclei in cloning efficiency: can the ooplasm reprogram any nucleus? Yoko Kato and Yukio Tsunoda Int. J. Dev. Biol. (2010) 54: 1623-1629 https://doi.org/10.1387/ijdb.103203yk

\section{Nuclear reprogramming in zygotes}

Chanchao Lorthongpanich, Davor Solter and Chin Yan Lim Int. J. Dev. Biol. (2010) 54: 1631-1640 https://doi.org/10.1387/ijdb.103201cl

Enhancing somatic nuclear reprogramming by Oct4 gain-of-function in cloned mouse embryos Martin J. Pfeiffer, Sebastian T. Balbach, Telma C. Esteves, Nicola Crosetto and Michele Boiani Int. J. Dev. Biol. (2010) 54: 1649-1657 https://doi.org/10.1387/ijdb.103197mp

\section{Nuclear reprogramming in zygotes}

Chanchao Lorthongpanich, Davor Solter and Chin Yan Lim Int. J. Dev. Biol. (2010) 54: 1631-1640 https://doi.org/10.1387/ijdb.103201cl

Cloning of non-human primates: the road "less traveled by" Michelle L. Sparman, Masahito Tachibana and Shoukhrat M. Mitalipov Int. J. Dev. Biol. (2010) 54: 1671-1678 https://doi.org/10.1387/ijdb.103196ms

The influence of donor nucleus source on the outcome of zebrafish somatic cell nuclear transfer

Kannika Siripattarapravat, Boonya Pinmee, Eun-Ah Chang, Juan D. Muñoz, Koichi Kawakami and José B. Cibelli

Int. J. Dev. Biol. (2010) 54: 1679-1683

https://doi.org/10.1387/ijdb.103189ks
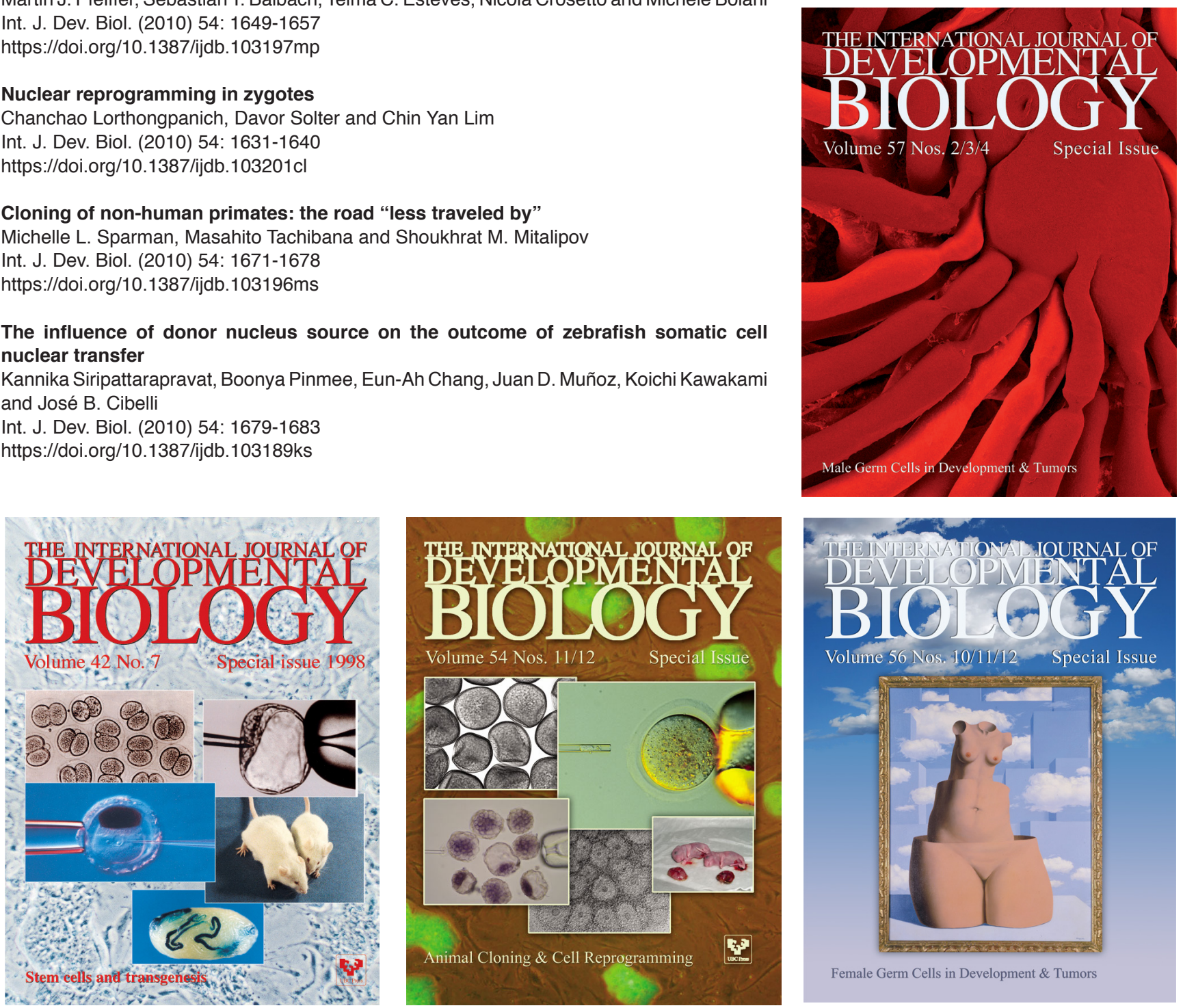